\title{
Small-Molecule Screens Reveal Novel Haustorium Inhibitors in the Root Parasitic Plant Triphysaria versicolor
}

\author{
Yaxin Wang, Daniel Steele, Maylin Murdock, Seigmund Lai, and John Yoder ${ }^{\dagger}$ \\ Department of Plant Sciences, University of California, Davis, CA \\ Accepted for publication 25 June 2019.
}

\begin{abstract}
Root parasitic weeds in Orobanchaceae pose a tremendous threat to agriculture worldwide. We used an in vitro assay to screen libraries of small molecules for those capable of inhibiting or enhancing haustorium development in the parasitic plant Triphysaria versicolor. Several redoxmodifying molecules and one structural analog of 2,6-dimethoxybenzoquine (DMBQ) inhibited haustorium development in the presence of the haustorium-inducing factor DMBQ, some of these without apparent

growth inhibition to the root. Triphysaria seedlings were able to acclimate to some of these redox inhibitors. Transcript levels of four early-stage haustorium genes were differentially influenced by inhibitors. These novel haustorium inhibitors highlight the importance of redox cycling for haustorium development and suggest the potential of controlling parasitic weeds by interrupting early-stage redox-signaling pathways.
\end{abstract}

Parasitic weeds are among the world's most economically damaging agricultural pests. The most destructive groups include witchweeds (genus Striga), broomrapes (genera Orobanche and Phelipanche), dodders (genus Cuscuta), and mistletoes (genera Viscum and Arceuthobium) (Parker 2012). The genera Striga, Orobanche, and Phelipanche are root parasite Orobanchaceae that attack a large number of crops, including corn, rice, sorghum, sunflower, and tomato (Parker 2012; Yoder and Scholes 2010). The tightly controlled subterranean interactions between hosts and parasitic plants make these weeds difficult to control, because the majority of damage occurs before the parasites emerge from the soil. Yield losses in infested Striga fields were reported to range from 40 to $90 \%$ (Aly 2007). Although progress has been made in reducing the damage caused by parasitic weeds through agronomic practices, genetically resistant crop varieties, and selective herbicides, effective and economically feasible approaches are still needed (Rubiales et al. 2009).

Parasitic plants use structures known as haustoria to attach and invade their host to obtain water and nutrients (Yoshida et al. 2016). Obligate root parasites, such as the genera Striga and Orobanche, form terminal haustoria at the tips of the emerging radical meristem (Nickrent et al. 1979; O'Malley and Lynn 2000). Facultative root parasites, such as the genera Triphysaria and Phtheirospermum, are nonweedy hemiparasites that develop lateral haustoria primarily in the elongation zone of root tips (Cui et al. 2015; Goldwasser et al. 2002; Nickrent et al. 1979).

Haustoria develop in response to haustorium-inducing factors (HIFs) released by neighboring host roots (Albrecht et al. 1999). Quinones and related phenols have been identified as active HIFs (Albrecht et al. 1999; Cui et al. 2018; Lynn et al. 1981; Steffens et al. 1982). The only HIF yet identified from host plants is 2,6dimethoxybenzoquinone (DMBQ), which was extracted from roots

†Corresponding author: J. Yoder; jiyoder@ucdavis.edu

Funding: This work was supported by National Science Foundation grants DBI0701748 and IOS-1238057. Y. Wang and D. Steele were supported by UC Davis Plant Sciences Department GSR and Henry A. Jastro Graduate Research Awards.

*The $\boldsymbol{e}$-Xtra logo stands for "electronic extra" and indicates that two supplementary figures, three supplementary tables, and two supplementary videos are published online.

The author(s) declare no conflict of interest.

(C) 2019 The American Phytopathological Society of sorghum (Chang and Lynn 1986). The evaluation of structurally similar quinones for their ability to induce Striga haustoria demonstrated that only those with redox potentials within a relatively narrow window were active (Keyes et al. 2000).

One potential approach for controlling parasitic weeds in the field would be to inhibit the development of haustoria. Intercropping susceptible host crop maize with the fodder legumes Desmodium uncinatum and Desmodium intortum significantly reduced Striga hermonthica infestation by inhibiting the primary haustoria growth (Khan et al. 2002). A genetic approach identified wild relatives of sorghum that displayed resistance to the genus Striga by reducing haustoria initiation (Rich et al. 2004). A transgenic approach to inhibiting functional haustorium attachment of dodder was achieved by expressing the inhibitive propeptide segment of a cysteine proteinase in host tomato plants (Amini et al. 2017).

Two chemical inhibitors of haustorium development in the genus Striga have been reported. Tetrafluorobenzoquinone (TFBQ) and cyclopropyl-p-benzoquinone (CPBQ) are structurally similar to DMBQ and possibly bind competitively with DMBQ receptors (Smith et al. 1996). TFBQ reversibly inhibited haustorium induction in the genus Striga, whereas CPBQ irreversibly inhibited induction in the genus Striga by undergoing irreversible ring opening through the semiquinone (Zeng et al. 1996). Studies with specific inhibitors of haustorium, TFBQ and $\mathrm{CPBQ}$, implicated that one-electron redox cycling between quinone and semiquinone states was critical for haustorium induction (Smith et al. 1996). This was later supported by the isolation and in planta characterization of a single-electron NADPH:quinone oxidoreductase gene, TvQR1, from Triphysaria versicolor (Matvienko et al. 2001). Inhibition of $T v Q R I$ transcription in Triphysaria roots by RNA interference (RNAi) resulted in a significant reduction of haustoria formation in response to DMBQ or host root exudates, whereas inhibition of the closely related two-electron reducing quinone oxidoreductase $T v Q R 2$ did not (Bandaranayake et al. 2010).

Other genes involved in early stages of haustorium development have been similarly functionally identified in Orobanchaceae. TvPirin encodes a transcription factor that, when inhibited in Triphysaria roots by $\mathrm{RNAi}$, reduces haustorium formation (Bandaranayake et al. 2012). PjYUC3, identified in Phetheirospermum japonicum, encodes a YUCCA flavin monooxygenase that is involved in auxin biosynthesis. The transcript level of PjYUC3 increases in response to HIF treatment, and reducing its level by RNAi reduces haustoria induction (Ishida et al. 2016). 
In this study, we screened two classes of molecules for their ability to reduce or enhance haustorium development in Triphysaria versicolor in the presence of DMBQ. One class was commercially available structural analogs of DMBQ that were selected on the possibility of them interacting directly with the HIF receptors. The other class of molecules examined was known disruptors of redoxsignaling pathways. Our research identified novel haustorium inhibitors and revealed the essential role of intracellular redox signaling in haustorium development in the genus Triphysaria.

\section{MATERIALS AND METHODS}

Plant materials and growth conditions. Seeds of the outcrossing species $T$. versicolor were collected from an open pollinated population growing in a pasture land south of Napa, California (global positioning system location: $38^{\circ} 13^{\prime} 33.2^{\prime \prime} \mathrm{N}$, $\left.122^{\circ} 16^{\prime} 11.7^{\prime \prime} \mathrm{W}\right)$. Triphysaria seeds were sterilized and germinated on $1 / 4$ Hoagland nutrient medium ( $\mathrm{pH} 6.1$ ) containing $0.75 \%$ (wt/ vol) Phytagel (P8169; Sigma) in a $16^{\circ} \mathrm{C}$ growth chamber under cycles of $12 \mathrm{~h}$ of light and $12 \mathrm{~h}$ of darkness (Jamison and Yoder 2001). Approximately 1 week after germination, Triphysaria seedlings were aseptically transferred to square petri dishes (25 to 50 seedlings per plate) with $25 \mathrm{ml}$ of $1 / 4$ Hoagland nutrient media (pH 6.1), $0.75 \%$ (wt/vol) sucrose, and $0.75 \%$ (wt/vol) Phytagel. The square petri dishes were placed vertically in $22^{\circ} \mathrm{C}$ growth chamber under cycles of $16 \mathrm{~h}$ of light and $8 \mathrm{~h}$ of darkness.

Reagents. The Screen-Well Redox Library (BML-2835-0500) was purchased from Enzo Life Sciences. The molecules are referred to in this paper by their positions in the 96-well tray that they came in, and the key to their names is given in Supplementary Tables S1 and S2. The molecules DMBQ (428566; Sigma), tetraethylthiuram disulfide (86720, $\geq 97.0 \%$; Sigma), TFBQ (104353; Sigma), tanshinone IIA (S2365; Selleck Chemical LLC; Fisher Scientific Company LLC), ebselen (E0946; TCI America; Spectrum Chemicals \& Laboratory Products), and $N$-ethylmaleimide (F11; AC156100050; Acros Organics, Fisher Scientific Company LLC) were purchased from the indicated vendors.

Screening of potential inhibitors and enhancers of haustorium development. Molecules in the SCREEN-WELL
Redox Library were originally shipped in as $10 \mathrm{mM}$ stock solutions in dimethyl sulfoxide (DMSO). The stock solutions of structural analogs of DMBQ were prepared by suspending to $10 \mathrm{mM}$ in DMSO. Each molecule was further diluted in water and mixed with DMBQ to reach a final concentration of $100 \mu \mathrm{M}$ test molecule and $3 \mu \mathrm{M}$ DMBQ.

Haustorium development was assayed as previously described (Jamison and Yoder 2001). Triphysaria seedlings were transferred from germination plates onto induction plates at least 2 days before DMBQ induction. One milliliter of the DMBQ and test molecule solution was added directly on top of Triphysaria root tips. Plates were kept horizontal for $2 \mathrm{~h}$ before returning to the growth chamber. The number of haustoria was determined $24 \mathrm{~h}$ after treatment using a dissecting microscope. Haustorium development was quantified as the proportion of plants that developed haustoria. Three to four biological replicates were performed for each molecule.

Root growth assay. Ten to fifteen 1-week-old Triphysaria seedlings were transferred onto square petri dishes containing $25 \mathrm{ml}$ of 1/4 Hoagland's nutrient media ( $\mathrm{pH} \mathrm{6.1),0.75 \%} \mathrm{(wt/vol)} \mathrm{sucrose,}$ and $0.75 \%$ (wt/vol) Phytagel. The square petri dishes were placed vertical in a $22^{\circ} \mathrm{C}$ growth chamber under cycles of $16 \mathrm{~h}$ of light and $8 \mathrm{~h}$ of darkness, and root growth was monitored by marking the plates at the position of the root tips every $24 \mathrm{~h}$. Three days after transfer, $1 \mathrm{ml}$ of inhibitor $(50 \mu \mathrm{M} \mathrm{F} 11,100 \mu \mathrm{M}$ C5, $100 \mu \mathrm{M}$ G5, $100 \mu \mathrm{M} F 1$, or $100 \mu \mathrm{M}$ TFBQ) was added to the plates. Plates were kept horizontal for $1 \mathrm{~h}$ to allow absorption of the inhibitor liquid into the media and then, returned vertically into the growth chamber. Root growth was monitored for an additional 5 days after the inhibitor treatment. Photos of the plates were taken, and daily root growth was measured using ImageJ $(\mathrm{NIH})$. For each inhibitor treatment, four plates with a total of 40 to 50 seedlings were measured.

Redox inhibitor acclimation assay. Inhibitors were added into the media before pouring the plates to reach a final concentration of $8 \mu \mathrm{M}$, which is equivalent to $2 \mathrm{ml}$ of $100 \mu \mathrm{M}$ inhibitor solution added to a $25-\mathrm{ml}$ media plate. Seedlings grown vertically for at least 2 days were transferred onto freshly prepared inhibitor plates and treated with $10 \mu \mathrm{M}$ DMBQ immediately (day 0 ) or 5 days later (day 5 with seedlings). A second set of freshly

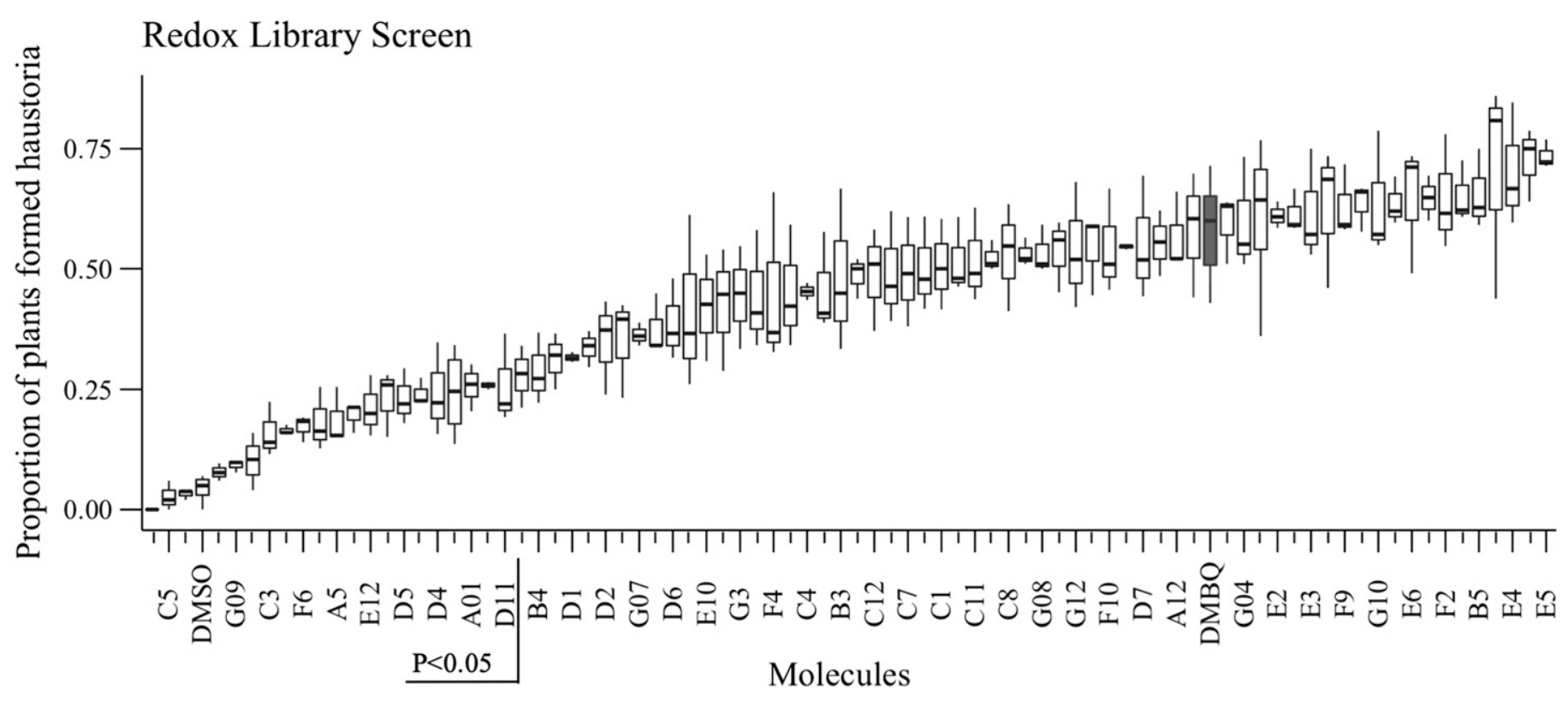

Fig. 1. Redox library screen for inhibitive or enhancing activities of molecules on haustorium development. Molecules from Screen-Well Redox Library (BML2835; Enzo Life Sciences) were screened at $100 \mu \mathrm{M}$ for their interactions with $3 \mu \mathrm{M}$ 2,6-dimethoxybenzoquine (DMBQ) on haustorium induction. The molecule identifier denotes the position of the inhibitor on the 96-well plate. Every other molecule ranking high to low in haustorium inhibition is labeled on the $x$ axis. Complete molecule ranking list is available. $P<0.05$ indicates molecules that show statistically significant inhibition of $3 \mu \mathrm{M}$ DMBQ by Tukey honestly significant difference test. DMBQ, positive control; DMSO, dimethyl sulfoxide, negative control. 
prepared inhibitor plates was placed in the same growth conditions without Triphysaria seedlings. After 5 days, Triphysaria seedlings growing on inhibitor-free plates were transferred onto the inhibitor plates and treated with $10 \mu \mathrm{M}$ DMBQ (day 5). The proportion of
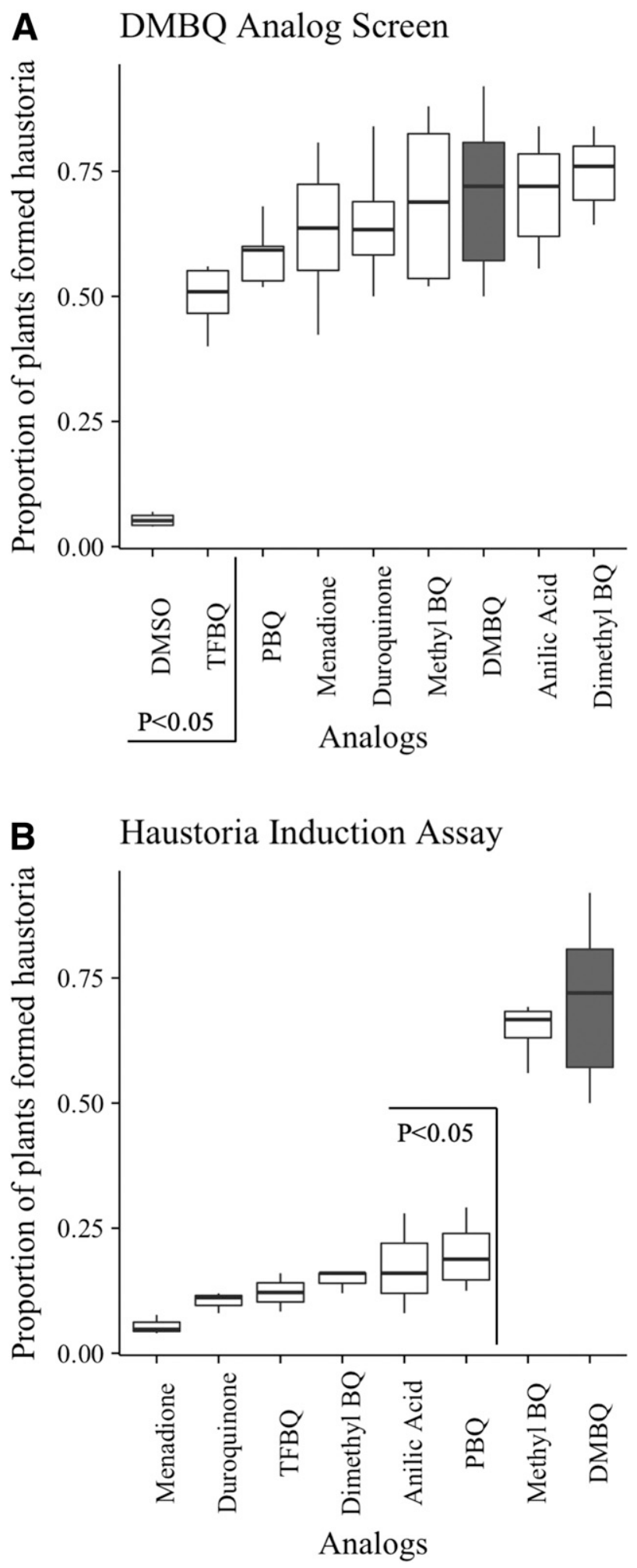

Fig. 2. The 2,6-dimethoxybenzoquine (DMBQ) analog library screen. A, DMBQ analogs were screened at $100 \mu \mathrm{M}$ for their inhibitive or enhancing interaction with $3 \mu \mathrm{M}$ DMBQ on haustorium development. $P<0.05$ indicates the molecules that show statistically significant inhibition of DMBQ induction by Tukey HSD test. B, Haustorium-inducing activity of DMBQ analogs at $100 \mu \mathrm{M} . P<0.05$ indicates the molecules that show statistically significant difference from $3 \mu \mathrm{M}$ DMBQ induction by Tukey HSD test. TFBQ, tetrafluorobenzoquinon; $\mathrm{PBQ}$, 2-Phenyl-1,4-benzoquinone; and $\mathrm{BQ}$, benzoquinone. plants that developed haustoria was counted $24 \mathrm{~h}$ later under a dissecting microscope.

Transcriptional analysis. Transcript levels of four Triphysaria genes associated with early haustorium development (TvQRI, $T v Q R 2, T v$ Pirin, and TvYUC9) were determined by quantitative real-time PCR in the presence and absence of inhibitors (Bandaranayake et al. 2010, 2012; Ishida et al. 2016). A Triphysaria transcript homologous to the PjYUC3 sequence (Ishida et al. 2016) was identified by BLAST homology searches against the PPGP transcriptome database (http://ppgp.huck.psu.edu/) (Yang et al. 2014). Because the $Y U C$ gene identified in the genus Triphysaria that showed upregulation during early haustorium development was most closely related to the AtYUC9 in the genus Arabidopsis, we named it TVYUC9.

Triphysaria root tips were harvested $1 \mathrm{~h}$ after treatment with redox inhibitors with and without DMBQ or DMBQ analogs with and without $\mathrm{DMBQ}$, and they were frozen in liquid nitrogen. The tissue was ground in liquid nitrogen, and RNA was isolated using the RNeasy Plant Mini Kit (Qiagen). RNA was treated with DNase I before reverse transcription. One microgram or less of RNA was converted to cDNA using the High-Capacity cDNA Reverse Transcription Kit (Applied Biosystems). Each cDNA was diluted 50-fold and used for SYBR green-based real-time quantitative PCR assays in the ABI 7300 quantitative PCR system (Applied Biosystems).

Primer pairs for quantitative PCR were validated by building standard curves with four 10-fold serial dilutions. Only primer pairs that showed $>90 \%$ amplification efficiency and generated a single peak in the dissociation curve were selected (Sigma-Aldrich 2012). Transcript levels were measured in three technical replicates for each of three to four biological replicates. Gene expression levels were calculated using the $\Delta \Delta \mathrm{Ct}$ (cycle threshold) method relative to the geometric mean of two reference genes TvQNA 8 and TvTUB1 (Vandesompele et al. 2002). The primers used are listed in Supplementary Table S3.

Statistical analysis. All of the experiments were completed with at least three biological replicates. Statistical analysis was carried out in R. Analysis of variance for experiments with more than two treatments were carried out by Tukey HSD (honestly significant difference) test. Trend analysis for inhibitor and inducer competition assay was carried out by fitting the DMBQ levels in a linear model twice: once as a covariate factor (as numeric) and once as a categorical factor (as factor). Significant $P$ value for covariate effect indicates that there is a significant nonzero linear component in the model. This analysis showed the linear trend of a model without having to assume the specific form of the model.

\section{RESULTS}

Chemical screening identified inhibitors, but no enhancers, of haustorium development. We evaluated 84 different pro- and antioxidant molecules available in the Screen-Well Redox Library (BML-2835; Enzo Life Science) as well as nine structural analogs of DMBQ for their ability to inhibit or promote haustorium development in the presence of DMBQ. Each molecule was diluted to $100 \mu \mathrm{M}$ and combined with $3 \mu \mathrm{M}$ DMBQ before adding to roots of Triphysaria seedlings. A suboptimal concentration of DMBQ was used to induce about $50 \%$ of the plants to form haustoria so that both inhibitive and enhancing activities of the screened molecule would be detected (Jamison and Yoder 2001). Two molecules (A3 and B12) in the redox library and two molecules (1,4-naphthoquinone and juglone) in the DMBQ analog library caused the Triphysaria roots to brown and die; therefore, these were eliminated from additional analyses.

As shown in Figure 1, 22 molecules from the redox library showed significant reduction of haustorium development based on Tukey HSD test with a $P$ value $<0.05$, whereas none of the molecules significantly enhanced haustorium development. Several additional redox molecules inhibited haustorium, although not to the 
selected statistical significance levels (Fig. 1). Only one structural analog of DMBQ, TFBQ, reduced haustorium development to the statistical significance cutoff, although to a lesser degree than the
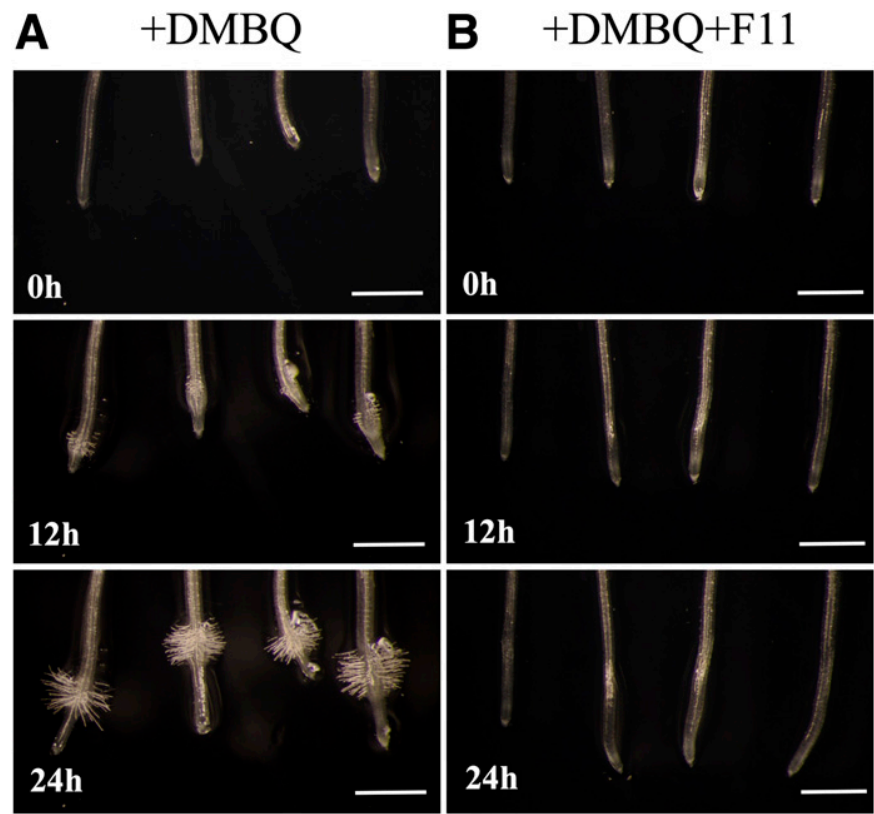

Fig. 3. Inhibition of haustorium development by redox inhibitor $N$-ethylmaleimide (F11). A, The $10 \mu \mathrm{M}$ 2,6-dimethoxybenzoquine (DMBQ) induced Triphysaria seedlings to develop haustorium. B, The $50 \mu \mathrm{M}$ F11 combined with $10 \mu \mathrm{M}$ DMBQ inhibited haustorium initiation. Time-lapse videos are presented in Supplementary Videos S1 and S2. Scale bar, $1 \mathrm{~mm}$. most inhibitive redox molecules (Fig. 2A). Of seven DMBQ analogs examined, only 2-methyl-1,4-benzoquinone (methyl BQ) showed haustorium-inducing activity to the level similar to DMBQ induction (Fig. 2B). Figure 3 illustrates the inhibition of haustorium development by the most active inhibitor F11 identified in the small-molecule screens. The relatively large number of redox-modifying molecules that inhibited haustorium development highlights the importance of redox signaling in haustorium induction.

Because physical properties of chemicals may relate to their biological activities, we identified chemical and physical features of the molecules in the redox library and the DMBQ analog library by searching PubChem (https://pubchem.ncbi.nlm.nih.gov) and the (U.S. Environmental Protection Agency) chemistry dashboard (https://comptox.epa.gov/dashboard). To determine if certain physical properties were associated with haustorium inhibition, we analyzed the physical properties of the 10 most inhibitive (TOP10-Inhibitive) and 10 least inhibitive molecules (TOP10-Not Inhibitive) (Tables 1 and 2) from the redox library screen. The TOP10-Inhibitive molecules showed statistically significant lower total hydrogen bond counts (hydrogen donor and acceptor counts) and lower topological polarity surface areas compared with the TOP10-Not Inhibitive molecules (Fig. 4) of the 10 computed properties that we analyzed (Supplementary Fig. S1). The TOP10Inhibitive and TOP10-Not Inhibitive molecules have average hydrogen bond donor counts 0.6 and 3, respectively; average hydrogen bond acceptor counts of 2.5 and 4.8, respectively; and average polar surface areas of 50.97 and $95.79 \AA^{2}$, respectively. These values are consistent with the inhibitive molecules being highly permeable molecules and suggest that haustorium inhibition requires intracellular redox-state modification (Leeson and Springthorpe 2007; Lipinski 2004; Veber et al. 2002).

TABLE 1 . Top 10 most inhibitive molecules from redox library screen

\begin{tabular}{|c|c|c|c|c|c|}
\hline Structure & Name & Plate location & $\begin{array}{l}\text { Hydrogen bond } \\
\text { donor count }\end{array}$ & $\begin{array}{l}\text { Hydrogen bond } \\
\text { acceptor count }\end{array}$ & $\begin{array}{l}\text { Polar surface } \\
\text { area }\left(A^{2}\right)\end{array}$ \\
\hline & $N$-ethylmaleimide & $\mathrm{F}-11$ & 0 & 2 & 37.4 \\
\hline & Ebselen & C-05 & 0 & 1 & 20.3 \\
\hline & Disulfiram & G-05 & 0 & 4 & 121 \\
\hline & Tanshinone IIA & F-01 & 0 & 3 & 47.3 \\
\hline & Ethoxyquin & G-09 & 1 & 2 & 21.3 \\
\hline & Anethole trithione & G-01 & 0 & 4 & 91.9 \\
\hline & AA-861 & $\mathrm{C}-03$ & 1 & 3 & 54.4 \\
\hline & EPA & F-03 & 1 & 2 & 37.3 \\
\hline & Ibuproxam & F-06 & 2 & 2 & 49.3 \\
\hline & Cumene hydroperoxide & A-02 & 1 & 2 & 29.5 \\
\hline
\end{tabular}


Inhibitors showed different DMBQ concentration dependency on preventing haustorium development. The dose-responses of five inhibitors were further analyzed: TFBQ, F11 (N-ethylmaleimide), C5 (ebselen), G5 (disulfiram), and F1 (tanshinone IIA) (Table 1). In all cases, the inhibition of haustorium development was concentration dependent (Fig. 5A). F11 was the most potent haustorium inhibitor, reducing haustorium development $>90 \%$ at $50 \mu \mathrm{M}$. The other redox-modifying inhibitors (C5, $\mathrm{G} 5$, and F1) reduced haustorium development $90 \%$ at $100 \mu \mathrm{M}$. Therefore, in later assays, we applied $50 \mu \mathrm{M}$ F11 and $100 \mu \mathrm{M} \mathrm{C5}$, G5, and F1 to obtain sufficient haustorium inhibition. TFBQ reduced haustorium development $33 \%$ at $100 \mu \mathrm{M}$, and this concentration was used in later assays. TFBQ treatments at concentrations $>100 \mu \mathrm{M}$ caused Triphysaria roots to brown and die; therefore, they were avoided.

To determine the interactions of inhibitor molecules with DMBQ, we assayed whether the haustorium inhibition by selected inhibitors was dependent on DMBQ concentrations. Haustorium-inducing activities realized by increasing amount of DMBQ in the presence of a fixed amount of inhibitor were measured. Figure 5B showed that, in the DMSO-negative control when increasing DMBQ concentration from 3 to $30 \mu \mathrm{M}$, haustorium formation rates increased and peaked at $10 \mu \mathrm{M}$. A linear model was fitted to test the association between haustorium induction and DMBQ concentration (Supplementary Fig. S2), and significant covariate effect with a positive slope estimate indicates an association between haustorium induction and increasing DMBQ concentrations. DMSO control showed a significant linear component in the model of haustorium induction and DMBQ levels. In the presence of F11 and TFBQ, the models also showed a significant linear component, suggesting that increased concentration of DMBQ was able to recover haustorium formation (Fig. 5B). In contrast, the inhibitive activities of C5, G5, and F1 were not reduced by additional DMBQ. These results demonstrated that the haustorium inhibition activities of TFBQ and F11 were dependent on DMBQ concentration, whereas the other redox-modifying molecules inhibited haustorium development irrespective of DMBQ concentration. F11 and TFBQ might compete directly with DMBQ and block haustorium development. TFBQ has to be applied at the concentration about one magnitude higher than DMBQ to show significant inhibition, suggesting a not efficient competition that could be explained by the low affinity of TFBQ with DMBQ receptor.

Some molecules inhibited haustorium development but not root growth. We measured root growth before and after inhibitor treatment to determine the degree to which selected inhibitors affected root growth. As shown in Figure 6, TFBQ, F11, C5, and F1 were not observed to inhibit Triphysaria root growth. These haustorium inhibitors were, therefore, likely to function more specifically on haustorium development rather than general root growth. In contrast, G5 inhibited Triphysaria root growth for 2 days after treatment, but then, the roots resumed their pretreatment growth rates. Interestingly, growth inhibition was not observed until $24 \mathrm{~h}$ after exposure to G5, well after the period of haustorium development.

Triphysaria seedlings acclimate to redox disruption. We next asked whether the temporal nature of G5 growth inhibition was

TABLE 2. Top 10 least inhibitive molecules from redox library screen

\begin{tabular}{|c|c|c|c|c|c|}
\hline Structure & Name & Plate location & $\begin{array}{l}\text { Hydrogen bond } \\
\text { donor count }\end{array}$ & $\begin{array}{l}\text { Hydrogen bond } \\
\text { acceptor count }\end{array}$ & $\begin{array}{c}\text { Polar surface } \\
\text { area }\left(A^{2}\right)\end{array}$ \\
\hline & $N$-acetyl-cysteine & E-01 & 3 & 4 & 67.4 \\
\hline & $n$-octyl caffeate & E-06 & 2 & 4 & 66.8 \\
\hline & Gentisic acid & $\mathrm{C}-10$ & 3 & 4 & 77.8 \\
\hline & Probucol & F-02 & 2 & 4 & 91.1 \\
\hline & L-ergothioneine & B-02 & 2 & 3 & 96.3 \\
\hline & Procysteine & B-05 & 2 & 4 & 91.7 \\
\hline & Glutathione & E-09 & 6 & 8 & 160 \\
\hline & Ascorbic acid & E-04 & 4 & 6 & 107 \\
\hline & Ciclopirox ethanolamine & F-07 & 3 & 4 & 86.8 \\
\hline & Ascorbyl palmitate & E-05 & 3 & 7 & 113 \\
\hline
\end{tabular}


owing to acclimation of the genus Triphysaria to redox inhibitors. To test this, we grew seedlings on phytogel plates containing the inhibitor for 5 days before haustorium induction and compared haustorium development with seedlings freshly transferred onto similarly aged inhibitor plates. As shown in Figure 7, F11 and G5 lost most of their inhibitive activity after 5 days in phytogel, showing that they were unstable under plant culture conditions (day 0 compared with day 5). In contrast, although C5 and F1 did lose some inhibitive activity over time, they both retained at least some inhibitive activity after 5 days (day 0 compared with day 5). Importantly, Triphysaria seedlings that were maintained on C5 and F1 inhibitor plates for 5 days before DMBQ exposure had higher haustorium induction rates than seedlings transferred from plates without inhibitors (day 5 compared with day 5 with seedlings). These results indicated that Triphysaria seedlings were able to acclimate to the redox state fashioned by the inhibitors and partially recovered their ability to develop haustoria even in the presence of active inhibitors. An alternative explanation to this result was that Triphysaria seedlings might be able to degrade or metabolize C5 and F1 to a lower level; therefore, this led to an increased recovery of haustorium development.

Inhibitors differentially influence transcript levels of early-stage haustorium genes. We evaluated the effects of both redox inhibitors and DMBQ analogs on the steady-state transcript levels of four genes associated with early haustorium development: $T v Q R 1, T v Q R 2, T v P i r i n$, and the Triphysaria homolog of PjYUC3 named TVYUC9. We treated Triphysaria seedlings with one of four redox inhibitors or one of four DMBQ analogs with and without DMBQ. Messenger RNA was isolated after $1 \mathrm{~h}$ treatment of DMBQ, and quantitative real-time PCR was performed to determine transcript levels of each gene. Relative expressions of each gene were calculated based on geometric means of two reference genes: $T v T U B 1$ and $T v Q N A 8$. All treatments were normalized to DMSO treatment as control. As shown in Figure 8, DMBQ treatment increased the transcript levels of all genes from 5 to 30 times over mock treatments (with DMSO), consistent with previous findings (Bandaranayake et al. 2010, 2012; Ishida et al. 2016). The structural analogs of DMBQ induced transcript levels of the target genes from 2- to 70-fold compared with mock treatments. In contrast, the four redox inhibitors did not induce expression of TvPirin, $T v Y U C 9$, or $T v Q R 2$ but did generally increase the levels of $T v Q R 1$.

The redox inhibitors and structural analogs showed different effects on haustorium gene expression in the presence of DMBQ. The combination of DMBQ with most of the structural analogs increased the transcript level of early haustorium genes, whereas the combination of DMBQ with redox-associated inhibitors in general did not. However, there were some exceptions: F11 inhibited the upregulation of TvPirin and TvQR2 by DMBQ, F1 inhibited the upregulation of TvYUC 9 by DMBQ, and all molecules except F11 increased the expression of $T v Q R 1$. These results revealed at the molecular level that redox-modifying inhibitors and DMBQ analogs altered genes associated with haustorium development in distinguishing ways.

\section{DISCUSSION}

A large number of molecules from the redox library inhibited haustorium development, indicative of the importance of cellular redox state for this developmental process. Consistent with our findings, inhibitors of reactive oxygen species (ROS) and ROSregulating enzymes were reported to inhibit haustorium formation in S. hermonthica (Wada et al. 2019). Redox regulation and redoxrelated signaling pathways are involved in most processes in plant growth, development, and stress response (Considine and Foyer 2014; Dietz et al. 2016; Mhamdi and Van Breusegem 2018). Antioxidant redox-modifying molecules can directly scavenge ROS molecules, interact with cellular components, or upregulate antioxidant enzymes to influence cellular redox state (Das et al. 2015; Foyer and Noctor 2005; Noctor et al. 2018). Because of the broad effect of redox signaling in almost all development processes and the ability of parasitic plants to acclimate to or metabolize redox inhibitors, the application of redox inhibitors may not be a plausible method for parasitic weed control.

Comparative analysis of physical properties of the 10 most and 10 least inhibitive redox-modifying molecules revealed that active haustorium inhibitors had lower total hydrogen bond counts (hydrogen donor and acceptor counts) and lower topological polarity surface areas than inactive inhibitors. Low hydrogen bond donor and acceptor counts and reduced polar surface area are generally associated with high cell permeability and the bioavailability of drugs by oral consumption in clinical trials (Leeson and Springthorpe 2007; Lipinski 2004). The enrichment of highly permeable molecules being haustorium inhibitors suggests that the critical redox reactions associated with haustorium development are likely intracellular. Low cell permeability may be a significant factor to why several of the redox inhibitors screened did not show haustorium inhibition activities. It is worth noting that potential inhibitors were initially screened at only one concentration in our study. Clearly, molecules not showing inhibitive activities at this concentration may be able to inhibit haustorium at a higher concentration. For example, ascorbic acid at the concentration used in our study $(100 \mu \mathrm{M})$ was not inhibitive of haustorium induction in

\section{Hydrogen Bond Donor Count}
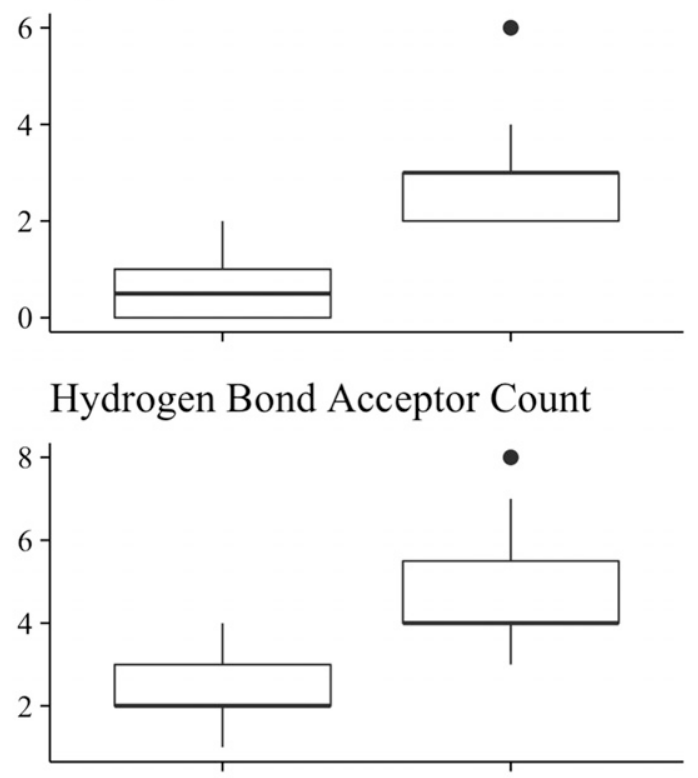

Polar Surface Area $\left(\AA^{\wedge} 2\right)$

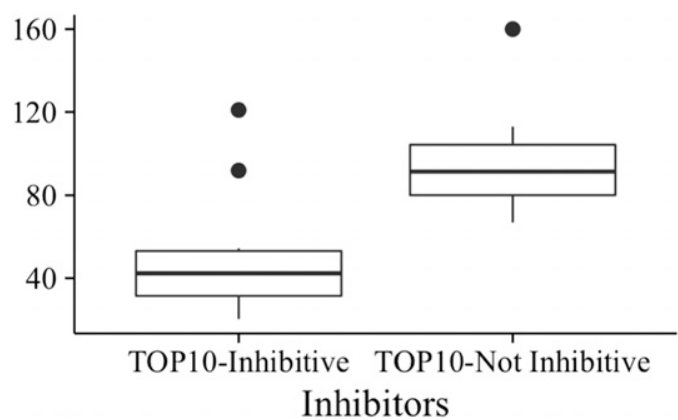

Fig. 4. Inhibitive and noninhibitive molecules from the redox library showed different chemical and physical properties. Comparison of computed properties of the 10 most inhibitive compounds (TOP10-Inhibitive) and the 10 least inhibitive compounds (TOP10-Not Inhibitive) in the redox library screen. 
either the genus Triphysaria (Table 2) or Striga (Wada et al. 2019), but it was inhibitive to haustorium formation in S. hermonthica when applied at a 10x higher concentration (1 mM) (Wada et al. 2019).

F11 is the most active haustorium inhibitor identified in this study. F11 is a thiol modifier that inhibits quinone-reducing activity of Arabidopsis P1-ZCr, the enzyme homologous to that encoded by TvQR1 in the genus Triphysaria (Matvienko et al. 2001; Rao et al. 1992). Additional mechanistic studies revealed that F11 inhibited at least one thiol group that contributed to the enzyme binding site with NADPH (Mano et al. 2000). Therefore, F11 may specifically reduce haustorium formation by trapping free thiols of NADPH: quinone oxidoreductases and thereby, inactivating their activities. Interestingly, NADPH oxidase inhibitors diphenyleneiodonium and phenylarsine oxide showed strong inhibition on haustorium formation in S. hermonthica (Wada et al. 2019).

Similar to F11, disulfiram (G5) can also irreversibly interact with thiols, forming covalent bonds with proteins at cysteine residues (Sakurai et al. 2006; Ying et al. 2007). Treatment of Triphysaria seedlings with disulfiram temporarily inhibited Triphysaria root growth but almost completely inhibited haustorium development. Similarly, tobacco seedlings growing on disulfiram media showed retarded but viable root growth, whereas pollen germination was totally inhibited (Op Den Camp and Kuhlemeier 1997). This can be explained by the fact that haustorium development and pollen growth are more sensitive to cellular redox states than root growth.

Plants acclimate to environmental cues through changing their redox states: for example, the acclimation to excessive light conditions during photosynthesis (Mhamdi and Van Breusegem 2018). Pretreatment of yeast cells with low concentrations of superoxide-generating agents, like hydrogen peroxide or menadione, induced adaptive detoxification and acclimation to oxidative stresses and protected cells from higher concentrations of oxidants in subsequent treatments (Jamieson 1992). Hydrogen peroxide pretreatment in Brassica napus led to increased activity of enzymatic and nonenzymatic antioxidants and enhanced glyoxalase system, and as a result, it prepared the seedlings with better defense against cadmium-induced oxidative stress (Hasanuzzaman et al. 2017). Triphysaria seedlings pretreated with redox inhibitors

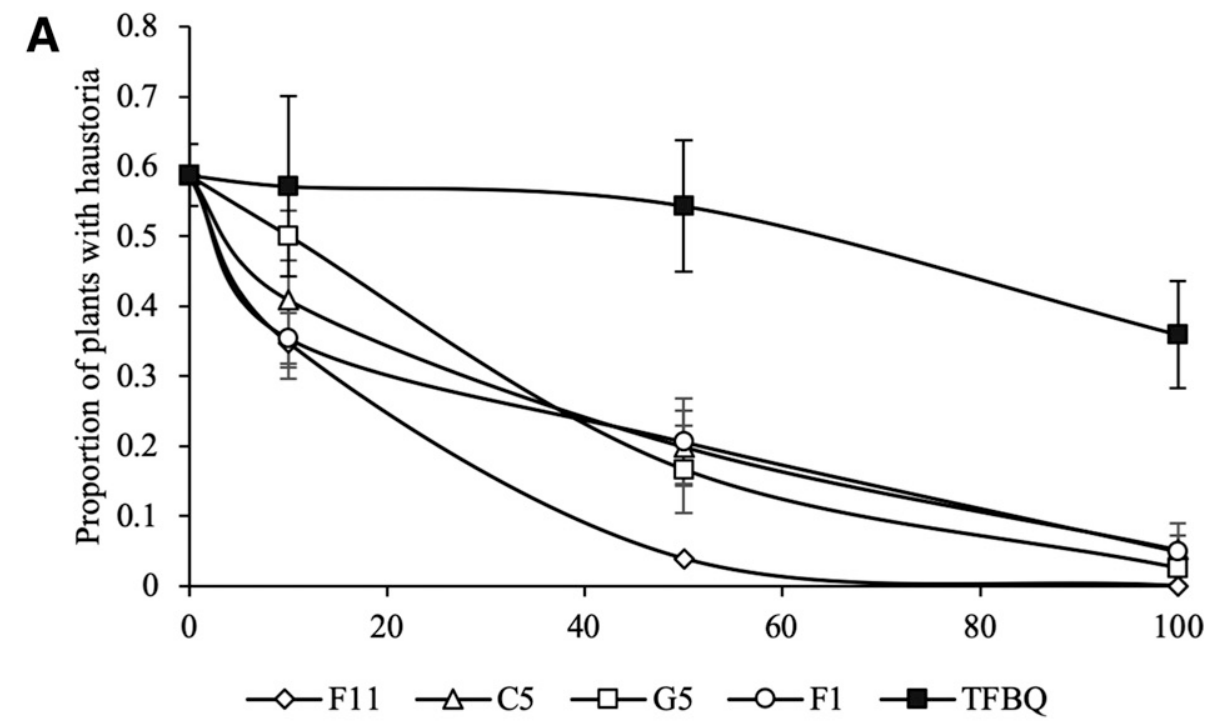

B

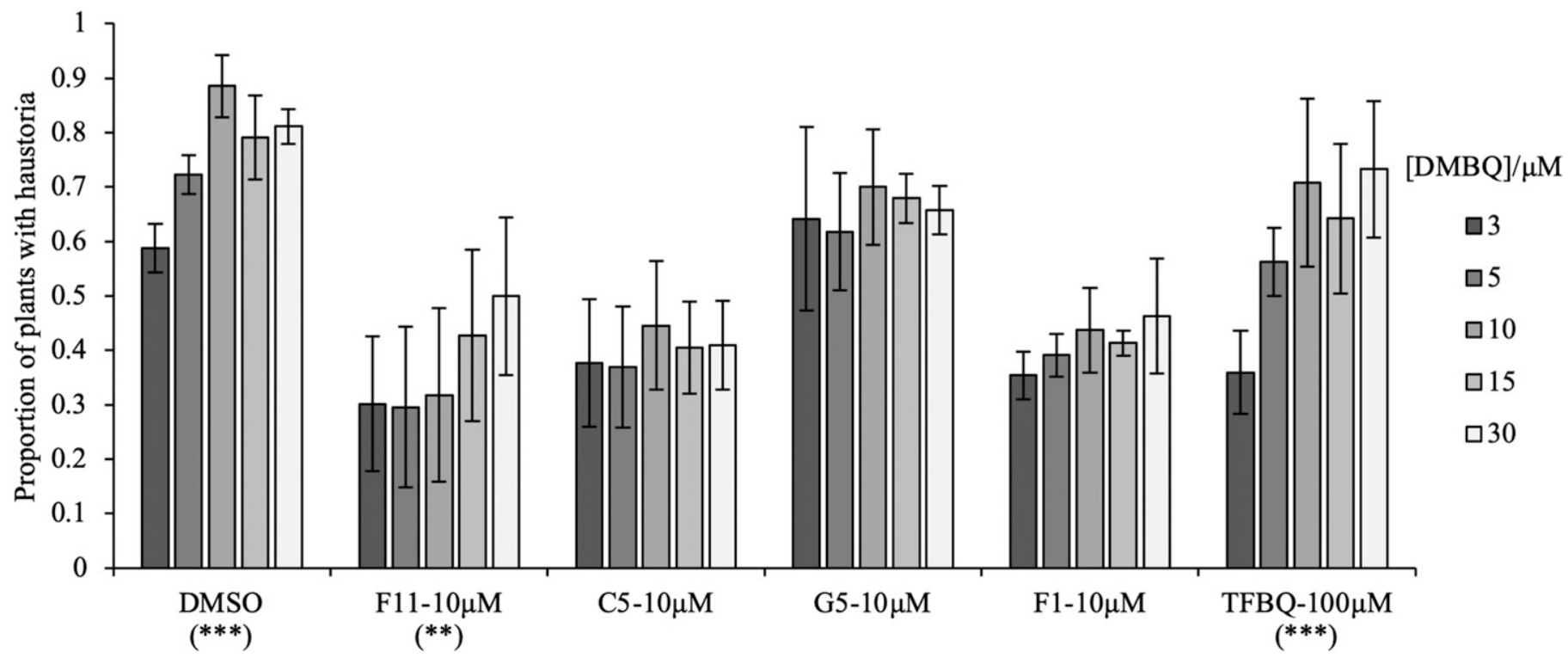

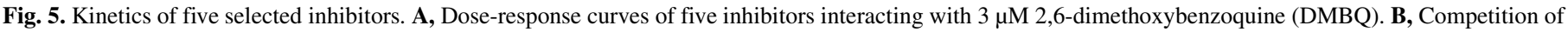

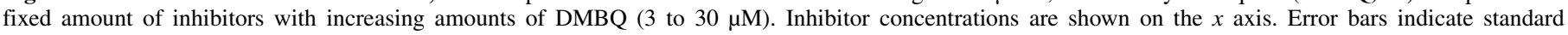

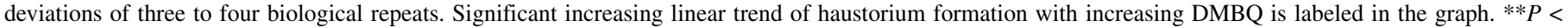
$0.01 ; * * * P<0.001$. 
perhaps acclimated in a similar mechanism by enhancing the antioxidant defense system and resuming pro- and antioxidant homeostasis, thus recovering the haustorium development capacity.

The redox-associated inhibitors that we identified were more active in preventing haustorium development compared with the DMBQ structural analogs. Of the nine structural analogs examined, only TFBQ significantly inhibited haustorium development. Increasing the concentration of DMBQ reduced haustorium inhibitive effects by TFBQ, which is consistent with the previous report that TFBQ reversibly inhibited haustorium induction in the genus Striga (Smith et al. 1996); it suggests that TFBQ might be a competitive inhibitor of DMBQ receptors in the genus Triphysaria.

TFBQ was first reported as a haustorium inhibitor for the genus Striga, where it is a much more potent inhibitor than in the genus Triphysaria; in the genus Striga, half-maximal inhibition occurred at $0.1 \mu \mathrm{M}$ (Smith et al. 1996), whereas significant inhibition (of 33\%) was observed at $100 \mu \mathrm{M}$ in the genus Triphysaria. In addition, hydroquinone (plate location: A-05) did not block induction by DMBQ in the genus Striga (Smith et al. 1996), but it significantly inhibited haustorium induction by DMBQ in the genus Triphysaria. These results highlight the differences in HIF recognition and haustorium development observed between different Orobanchaceae species.

Structural analogs of DMBQ induced the transcription of genes associated with early haustorium development without necessarily activating the haustorium development signaling pathway, except for methyl BQ. In contrast, the only haustorium gene transcriptionally induced by redox inhibitors in the absence of DMBQ was $T v Q R 1$. TvQR1 and TvQR2 are primary response genes that are induced by intrinsic or extrinsic signals independent of de novo protein synthesis of regulators (Bandaranayake et al. 2010; Matvienko et al. 2001). Redox inhibitors were able to induce the expression of $T v Q R 1$ but not $T v Q R 2$, suggesting that regulation of $T v Q R 1$ is redox sensitive. However, upregulation of early haustorium genes by structural analogs was not sufficient for haustorium development. Previously, it has been shown that menadione was able to induce the expression of $T v Q R 1$ and $T v Q R 2$ and that juglone induced high expression of $T v Q R 1$ (Matvienko et al. 2001). Both menadione and juglone are not haustorium inducers. Comparative transcriptome analysis of parasitism genes in three Orobanchaceae species suggests that genes involved in haustorium development have generally evolved from existing genes in nonparasitic angiosperms through neofunctionalization (Yang et al. 2014). The induction of haustorium genes by non-HIF compounds suggests that haustorium genes may fulfill functions in parasitic plants in addition to haustorium development.

This study identified novel haustorium inhibitors and showed that interrupting early-stages redox-signaling pathways has essential impact on haustorium development. These inhibitor molecules may function in other root parasitic plants as well that respond to similar

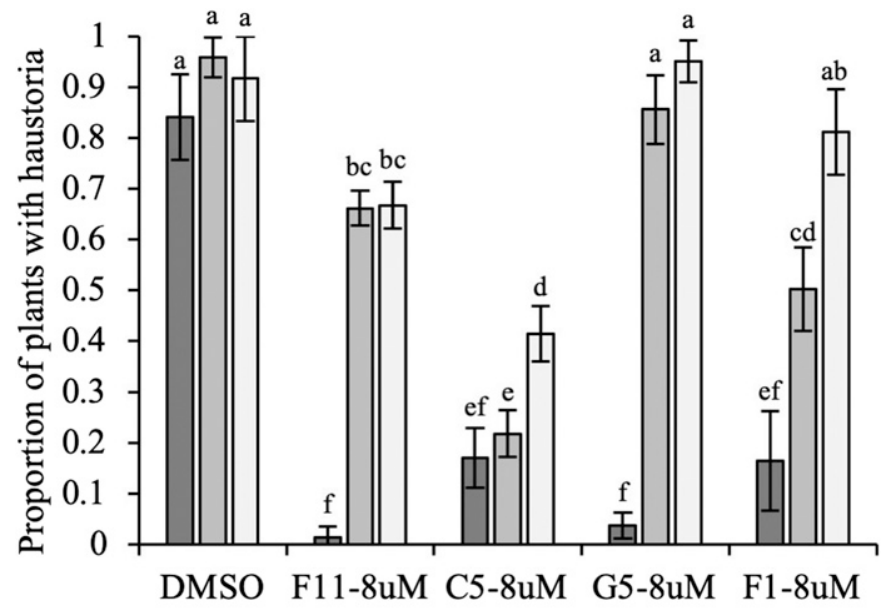

口Day0 aDay5 aDay5 w/seedlings

Fig. 7. Acclimation test on redox inhibitors. Haustoria induction rates were assayed by applying $10 \mu \mathrm{M}$ 2,6-dimethoxybenzoquine (DMBQ) to Triphysaria seedlings that were (i) transferred to the freshly prepared inhibitor plates (day 0 ), (ii) transferred to inhibitor plates that have been under the plant growth condition for 5 days (day 5), or (iii) kept growing on inhibitor plates for 5 days (day 5 with seedlings). Error bars indicate standard deviation of three to four biological replicates. Significant $(P<0.05)$ differences between means by Tukey honestly significant difference test are indicated by different letters. DMSO, dimethyl sulfoxide, negative control.

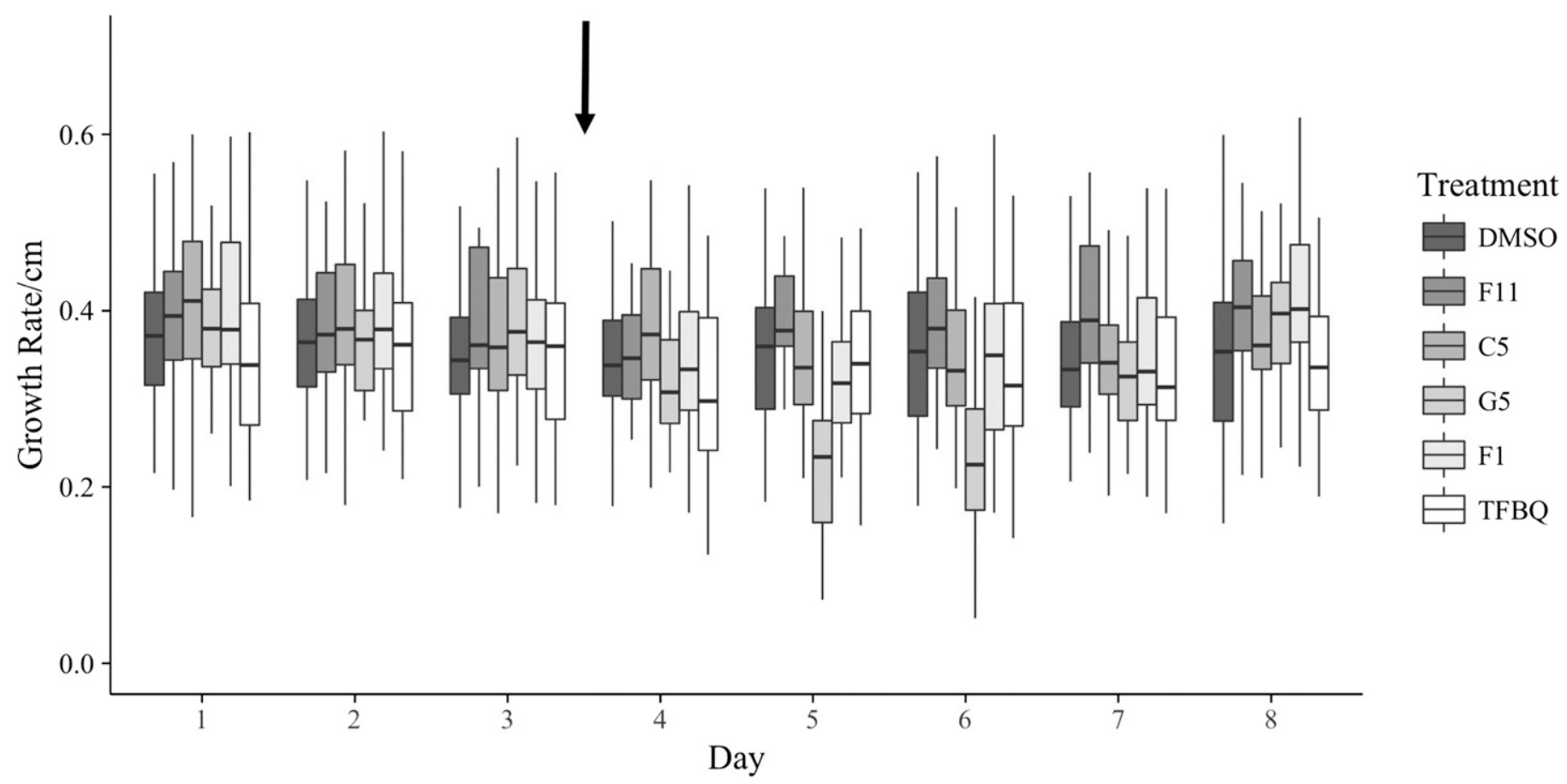

Fig. 6. Influence of five inhibitors on Triphysaria root growth. Inhibitor treatment was applied after day 3 measurement as indicated by the arrow. 

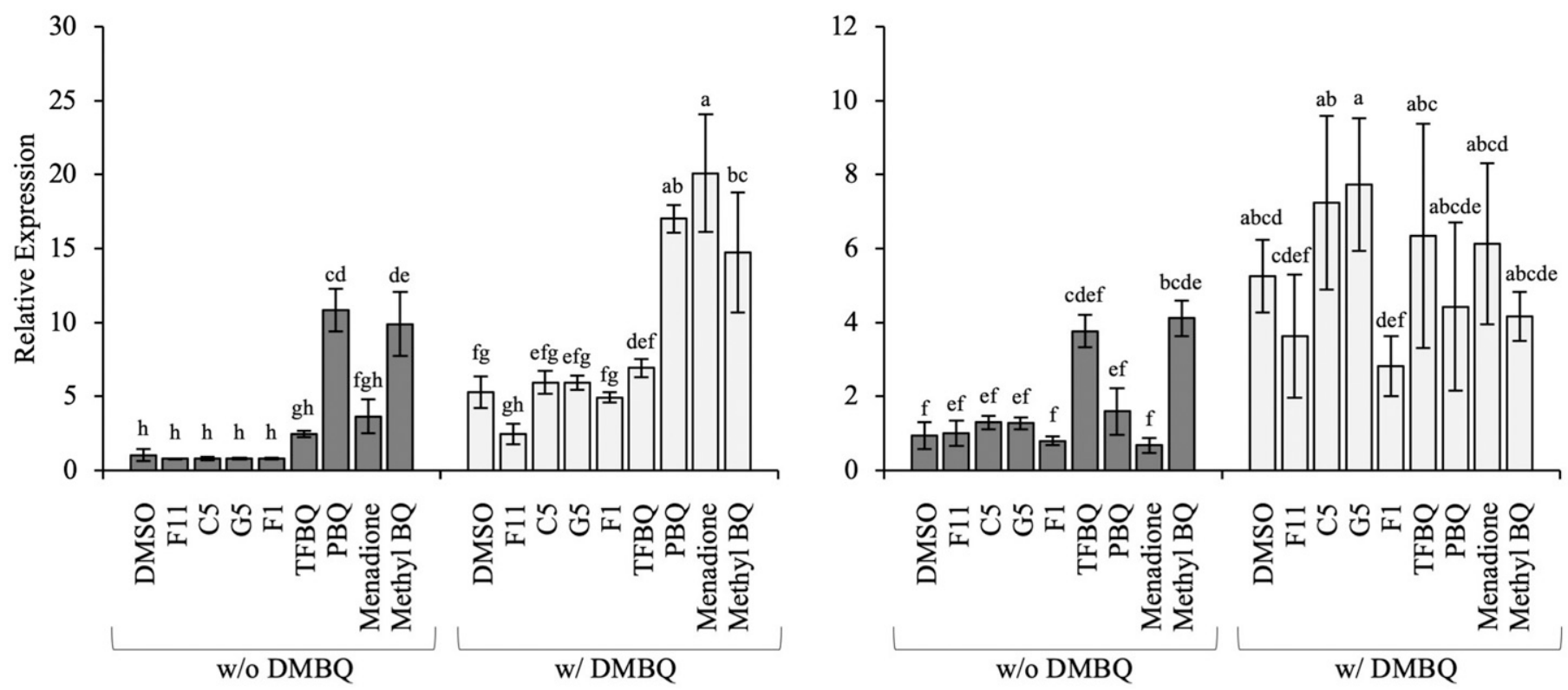

\section{TvQR2}
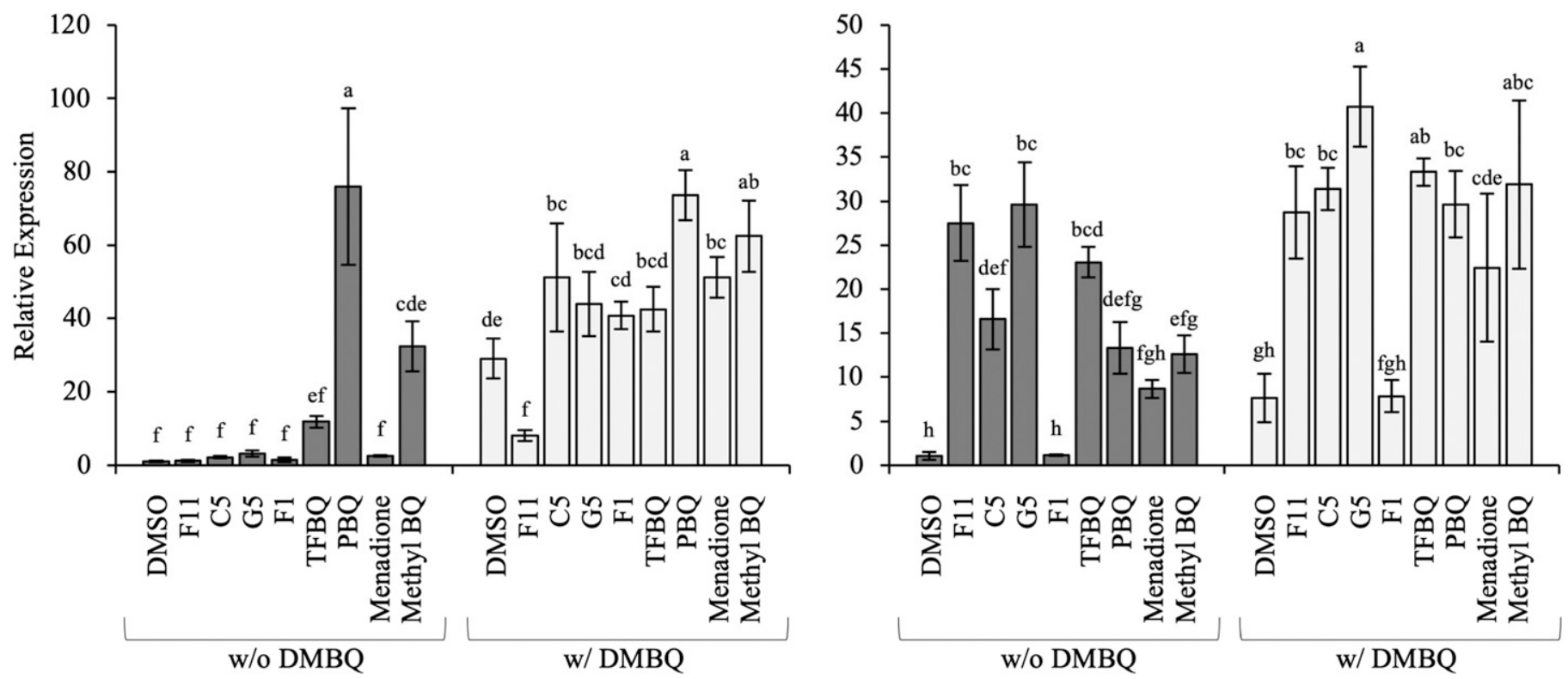

Fig. 8. Quantitative real-time PCR analysis of early haustorium genes after treatment of four redox inhibitors and four 2,6-dimethoxybenzoquine (DMBQ) analogs to Triphysaria seedlings. Relative expressions of each gene were calculated based on geometric mean of two reference genes: TvTUB1 and TvQNA8, and all were normalized to dimethyl sulfoxide (DMSO) treatment. Error bars indicate standard deviation of 3 to 10 biological replicates. Significant $(P<0.05)$ differences between means by Tukey's honestly significant difference test are indicated by different letters. With DMBQ, Triphysaria seedlings were treated with the test molecule in combination with $10 \mu \mathrm{M}$ DMBQ; without DMBQ, Triphysaria seedlings were treated only with test molecules (F11 at $50 \mu \mathrm{M}$ and the rest of molecules at $100 \mu \mathrm{M})$. TFBQ, tetrafluorobenzoquinon; PBQ, 2-Phenyl-1,4-benzoquinone; and BQ, benzoquinone.

types of quinone or phenolic HIFs through a redox-signaling pathway, and therefore, they can be utilized to extend the basic knowledge of haustorium development in parasitic plants. Additional research on specific control of redox reactions during haustorium development may provide potential control methods against parasitic weeds.

\section{ACKNOWLEDGMENTS}

We thank Neil Willits at the University of California, Davis Statistical Laboratory for the consultation of statistical analysis. We also thank
Florence Zakharov, Douglas Cook, members from the laboratory of J. Yoder, and colleagues on the Parasitic Plant Genome Project for thoughtful discussions and suggestions.

\section{LITERATURE CITED}

Albrecht, H., Yoder, J. I., and Phillips, D. A. 1999. Flavonoids promote haustoria formation in the root parasite Triphysaria versicolor. Plant Physiol. 119:585-592.

Aly, R. 2007. Conventional and biotechnological approaches for control of parasitic weeds. Vitr. Cell Dev. Biol. Plant. 43:304-317. 
Amini, M., Saify Nabiabad, H., and Deljou, A. 2017. Host-synthesized cysteine protease-specific inhibitor disrupts Cuscuta campestris parasitism in tomato. Plant Biotechnol. Rep. 11:289-298.

Bandaranayake, P. C. G., Tomilov, A., Tomilova, N. B., Ngo,Q., Wickett, N., dePamphilis, C. W., and Yoder, J. I. 2012. The TvPirin gene is necessary for haustorium development in the parasitic plant Triphysaria versicolor. Plant Physiol. 158:1046-1053.

Bandaranayake, P. C. G., Filappova, T., Tomilov, A., Tomilova, N. B., Jamison-McClung, D., Ngo, Q., Inoue, K., and Yoder, J. I. 2010. A singleelectron reducing quinone oxidoreductase is necessary to induce haustorium development in the root parasitic plant Triphysaria. Plant Cell 22:1404-1419.

Chang, M., and Lynn, D. G. 1986. The haustorium and the chemistry of host recognition in parasitic angiosperms. J. Chem. Ecol. 12:561-579.

Considine, M. J., and Foyer, C. H. 2014. Redox regulation of plant development. Antioxid. Redox Signal. 21:1305-1326.

Cui, S., Wada, S., Tobimatsu, Y., Takeda, Y., Saucet, S. B., Takano, T., Umezawa, T., Shirasu, K., and Yoshida, S. 2018. Host lignin composition affects haustorium induction in the parasitic plants Phtheirospermum japonicum and Striga hermonthica. New Phytol. 218:710-723.

Cui, S., Wakatake, T., Hashimoto, K., Saucet, S., Toyooka, K., Yoshida, S., and Shirasu, K. 2015. Haustorial hairs are specialized root hairs that support parasitism in the facultative parasitic plant, Phtheirospermum japonicum. Plant Physiol. 170:1492-1503.

Das, P., Nutan, K. K., Singla-pareek, S. L., and Pareek, A. 2015. Oxidative environment and redox homeostasis in plants: Dissecting out significant contribution of major cellular organelles. Front. Environ. Sci. 2:70.

Dietz, K.-J., Mittler, R., and Noctor, G. 2016. Recent progress in understanding the role of reactive oxygen species in plant cell signaling. Plant Physiol. 171:1535-1539.

Foyer, C. H., and Noctor, G. 2005. Redox homeostasis and antioxidant signaling: A metabolic interface between stress perception and physiological responses. Plant Cell 17:1866-1875.

Goldwasser, Y., Westwood, J. H., and Yoder, J. I. 2002. The use of arabidopsis to study interactions between parasitic angiosperms and their plant hosts. Arab. Book 1:e0035.

Hasanuzzaman, M., Nahar, K., Gill, S. S., Alharby, H. F., Razafindrabe, B. H. N., and Fujita, M. 2017. Hydrogen peroxide pretreatment mitigates cadmium-induced oxidative stress in Brassica napus L.: An intrinsic study on antioxidant defense and glyoxalase systems. Front. Plant Sci. 8:115.

Ishida, J. K., Wakatake, T., Yoshida, S., Takebayashi, Y., Kasahara, H., Wafula, E., dePamphilis, C. W., Namba, S. and Shirasu, K. 2016. Local auxin biosynthesis mediated by a YUCCA Flavin monooxygenase regulates haustorium development in the parasitic plant Phtheirospermum japonicum. Plant Cell 28:1795-1814.

Jamieson, D. J. 1992. Saccharomyces cerevisiae has distinct adaptive responses to both hydrogen peroxide and menadione. J. Bacteriol. 174: 6678-6681.

Jamison, D. S., and Yoder, J. I. 2001. Heritable variation in quinone-induced haustorium development in the parasitic plant Triphysaria. Plant Physiol. 125:1870-1879.

Keyes, W. J., O’Malley, R. C., Kim, D., and Lynn, D. G. 2000. Signaling organogenesis in parasitic angiosperms: Xenognosin generation, perception, and response. J. Plant Growth Regul. 19:217-231.

Khan, Z. R., Hassanali, A., Overholt, W., Khamis, T. M., Hooper, A. M., Pickett, J. A., Wadhams, L. J., and Woodcock, C. M. 2002. Control of witchweed Striga hermonthica by intercropping with Desmodium spp., and the mechanism defined as allelopathic. J. Chem. Ecol. 28:1871-1885.

Leeson, P. D., and Springthorpe, B. 2007. The influence of drug-like concepts on decision-making in medicinal chemistry. Nat. Rev. Drug Discov. 6:881-890.

Lipinski, C. A. 2004. Lead- and drug-like compounds: The rule-of-five revolution. Drug Discov. Today. Technol. 1:337-341.

Lynn, D. G., Steffens, J. G., Kamut, V. S., Graden, D. W., Shabanowitz, J., and Riopel, J. L. 1981. Isolation and characterization of the first host recognition substance for parasitic angiosperms. J. Am. Chem. Soc. 103: 1868-1870.

Mano, J., Babiychuk, E., Belles-Boix, E., Hiratake, J., Kimura, A., Inzé, D., Kushnir, S., and Asada, K. 2000. A novel NADPH:diamide oxidoreductase activity in Arabidopsis thaliana p1 $\zeta$-crystallin. Eur. J. Biochem. 267: 3661-3671.

Matvienko, M., Wojtowicz, A., Wrobel, R., Jamison, D., Goldwasser, Y., and Yoder, J. I. 2001. Quinone oxidoreductase message levels are differentially regulated in parasitic and non-parasitic plants exposed to allelopathic quinones. Plant J. 25:375-387.

Mhamdi, A., and Van Breusegem, F. 2018. Reactive oxygen species in plant development. Development 69:209-236.

Nickrent, D. L., Musselman, L. J., Riopel, J. L., and Eplee, R. E. 1979. Haustorial initiation and non-host penetration in witchweed (Striga asiatica). Ann. Bot. 43:233-236.

Noctor, G., Reichheld, J., and Foyer, C. H. 2018. ROS-related redox regulation and signaling in plants. Semin. Cell Dev. Biol. 80:3-12.

O'Malley, R. C., and Lynn, D. G. 2000. Expansin message regulation in parasitic angiosperms: Marking time in development. Plant Cell 12: 1455-1465.

Op Den Camp, R. G. L., and Kuhlemeier, C. 1997. Aldehyde dehydrogenase in tobacco pollen. Plant Mol. Biol. 35:355-365.

Parker, C. 2012. Parasitic weeds: A world challenge. Weed Sci. 60:269-276.

Rao, P. V., Krishna, C. M., and Zigler, J. S. 1992. Identification and characterization of the enzymatic activity of $\zeta$-crystallin from guinea pig lens: A novel NADPH:quinone oxidoreductase. J. Biol. Chem. 267: 96-102.

Rich, P. J., Grenier, C., and Ejeta, G. 2004. Striga resistance in the wild relatives of sorghum. Crop Sci. 44:2221-2229.

Rubiales, D., Verkleij, J., Vurro, M., Murdoch, A. J., and Joel, D. M. 2009. Parasitic plant management in sustainable agriculture. Weed Res. 49:1-5.

Sakurai, T., Kanayama, M., Shibata, T., Itoh, K., Kobayashi, A., Yamamoto, M., and Uchida, K. 2006. Ebselen, a seleno-organic antioxidant, as an electrophile. Chem. Res. Toxicol. 19:1196-1204.

Sigma-Aldrich. 2012. Assay Optimiztion and Validation. Pages 64-68 in: PCR Technologies, St. Louis, MO.

Smith, C. E., Ruttledge, T., Zeng, Z., O’Malley, R. C., and Lynn, D. G. 1996. A mechanism for inducing plant development: The genesis of a specific inhibitor. Proc. Natl. Acad. Sci. USA 93:6986-6991.

Steffens, J. C., Lynn, D. G., Kamut, V. S., and Riopel, J. L. 1982. Molecular specificity of haustorial induction in Agalinis purpurea (L.) Raf. (Scrophulariaceae). Ann. Bot. 50:1-7.

Vandesompele, J., De Preter, K., Poppe, B., Van Roy, N., De Paepe, A., and Speleman, F. 2002. Accurate normalization of real-time quantitative RTPCR data by geometric averaging of multiple internal control genes. Genome Biol. 3:research0034.1.

Veber, D. F., Johnson, S. R., Cheng, H. Y., Smith, B. R., Ward, K. W., and Kopple, K. D. 2002. Molecular properties that influence the oral bioavailability of drug candidates. J. Med. Chem. 45:2615-2623.

Wada, S., Cui, S., and Yoshida, S. 2019. Reactive oxygen species (ROS) generation is indispensable for haustorium formation of the root parasitic plant striga hermonthica. Front. Plant Sci. 10:328.

Yang, Z., Wafula, E. K., Honaas, L., Zhang, H., Das, M., Fernandez-Aparicio, M., Huang, K., Bandaranayake, P. C. G., Wu, B., Der, J. P., Clarke, C. R., Ralph, P. E., Landherr, L., Altman, N. S., Timko, M. P., Yoder, J. I., Westwood, J. H., and dePamphilis, C. W. 2014. Comparative transcriptome analyses reveal core parasitism genes and suggest gene duplication and repurposing as sources of structural novelty. Mol. Biol. Evol. 32:767-790.

Ying, J., Clavreul, N., Sethuraman, M., Adachi, T., and Cohen, R. A. 2007. Thiol oxidation in signaling and response to stress: Detection and quantification of physiological and pathophysiological thiol modifications. Free Radic. Biol. Med. 43:1099-1108.

Yoder, J. I., and Scholes, J. D. 2010. Host plant resistance to parasitic weeds; recent progress and bottlenecks. Curr. Opin. Plant Biol. 13: 478-484.

Yoshida, S., Cui, S., Ichihashi, Y., and Shirasu, K. 2016. The haustorium, a specialized invasive organ in parasitic plants. Annu. Rev. Plant Biol. 67: 643-667.

Zeng, Z., Cartwright, C. H., and Lynn, D. G. 1996. Chemistry of cyclopropylp-benzoquinone: A specific organogenesis inhibitor in plants. J. Am. Chem. Soc. 118:1233-1234. 